Meta

Journal des traducteurs

Translators' Journal

\title{
Stéréotypie, variants et invariants dans la traduction lexicographique des proverbes
}

\section{Mosbah Said}

Volume 53, numéro 2, juin 2008

La traduction des séquences figées

The Translation of Frozen Sequences

URI : https://id.erudit.org/iderudit/018520ar

DOI : https://doi.org/10.7202/018520ar

Aller au sommaire du numéro

Éditeur(s)

Les Presses de l'Université de Montréal

ISSN

0026-0452 (imprimé)

1492-1421 (numérique)

Découvrir la revue

Citer cet article

Said, M. (2008). Stéréotypie, variants et invariants dans la traduction lexicographique des proverbes. Meta, 53(2), 294-309.

https://doi.org/10.7202/018520ar
Résumé de l'article

La traduction des énoncés proverbiaux est souvent étudiée en rapport avec les écarts culturels entre les univers linguistiques. Or, la pluralité des équivalents d'un même proverbe dans les dictionnaires bilingues offre une occasion exceptionnelle pour analyser les variants et les invariants des représentations stéréotypiques associées à un tel énoncé à partir de la diversité actancielle et structurelle des équivalents qui lui sont relatifs. Ce regard pluriel de l'extérieur permettrait également de mieux saisir les enjeux de l'articulation entre la structure discursive et la structure logique du proverbe. 


\title{
Stéréotypie, variants et invariants dans la traduction lexicographique des proverbes
}

\author{
MOSBAH SAID \\ Université Tunis Carthage, Tunis, Tunisie \\ said_m_tn@yahoo.fr
}

\begin{abstract}
RÉSUMÉ
La traduction des énoncés proverbiaux est souvent étudiée en rapport avec les écarts culturels entre les univers linguistiques. Or, la pluralité des équivalents d'un même proverbe dans les dictionnaires bilingues offre une occasion exceptionnelle pour analyser les variants et les invariants des représentations stéréotypiques associées à un tel énoncé à partir de la diversité actancielle et structurelle des équivalents qui lui sont relatifs. Ce regard pluriel de l'extérieur permettrait également de mieux saisir les enjeux de l'articulation entre la structure discursive et la structure logique du proverbe.
\end{abstract}

\begin{abstract}
The translation of the proverbial statement is often examined in terms of the cultural distance between the linguistic universes. However, the various equivalents of the same proverb in bilingual dictionaries offer an exceptional opportunity to analyze variants and invariants of the stereotypic representations of the given statement based on the "actancial" and structural variety of its equivalents.

These alternative perspectives allow for a clearer understanding of the interplay between the proverb's discursive structure and its logical structure.
\end{abstract}

\section{MOTS-CLÉS/KEYWORDS}

arabe, dictionnaires bilingues, énoncés proverbiaux, stéréotypie, structure discursive et logique

\section{Introduction}

Le rapprochement entre stéréotypie et énoncé proverbial est parfois envisagé surtout en rapport avec le caractère figé de ce dernier en tant que "formule bien frappée» (Schapira 1999: 48), "phrase toute faite» ou stéréotype de pensée doté d'une valeur stylistique expressive. Autrement dit, ce qui est mis en relation dans ce rapprochement, c'est, d'une part, l'origine typographique qui voit dans toute expression toute faite un stéréotype et, d'autre part, le caractère imagé et l'expressivité incantatoire du proverbe consacrés entre autres par l'usage et la répétition.

$\mathrm{Au}$-delà de cet aspect nécessaire mais non suffisant, si nous considérons la stéréotypie comme un écart entre la représentation collective, instituée dans la langue, d'un fait, un objet, un être ou une situation et ce à quoi elle renvoie ou ce à quoi elle peut renvoyer dans la réalité, la stéréotypicalité du proverbe est alors à appréhender en rapport avec son sémantisme. Or, se présentant comme un truisme ou une vérité générale fondamentale, le contenu sémantique du proverbe admet, à première vue, difficilement cette notion d'écart dont l'étude dans le cadre d'un seul univers de croyance n'en ressort pas toute la pertinence. À cet égard, l'approche contrastive semble être un moyen efficace pour démystifier le caractère gnomique du proverbe 
et ce, en dévoilant certains de ses aspects sémantiques que laisseraient paraître sa confrontation avec des équivalents appartenant à d'autres univers linguistiques.

Dans la tradition lexicographique bilingue, le critère thématique joue souvent un rôle important dans la recherche des équivalences. Pour des dictionnaires différents traitant des mêmes langues, ce choix aboutit le plus souvent à l'établissement de plusieurs équivalents pour un même proverbe de la langue source comme c'est le cas dans les dictionnaires bilingues (français-arabe et arabe-français ${ }^{1}$ ) qui nous serviront d'illustration. Généralement établis dans l'esprit de l'équivalence fonctionnelle où l'invariant sémantique est déterminant dans leur choix, ces équivalents présentent presque toujours des variations actancielles par rapport à l'énoncé proverbial source conformément à l'esprit de chaque langue qui découpe la réalité à sa manière et érige des prototypes ou des images traduisant la différence d'ordre environnemental ou empirique. Dès lors, il est légitime de se demander où se situe l'essentiel de la charge stéréotypique dans le sens proverbial: se déploie-elle au niveau de l'invariant sémantique de cet énoncé ou au niveau de sa consécration de certains protagonistes et non d'autres? Autrement dit, dans le cas d'une diversité d'équivalents rattachés à un même proverbe en L1 sur la base d'une même thématique, la stéréotypie tient-elle à une simple variation de la catégorisation des objets et des êtres du monde au sein d'une même langue ou d'une langue à une autre ou résulte-t-elle plutôt d'un mécanisme plus profond qui implique une différence des moules de pensée ou du moins des univers de croyance?

$\mathrm{Au}$-delà de cette opposition relative, c'est le sens même du proverbe qui se trouve questionné. Faut-il l'appréhender au niveau de ce que rend la structure phrastique ou à un niveau, plus général, où il est question de sa structure propositionnelle logique?

Certes, le moyen le plus sûr pour répondre à cette question est de vérifier la synonymie entre les équivalents et le proverbe original à partir d'emplois précis. En cas de synonymie totale, celui-ci figurerait dans les mêmes contextes possibles que ses équivalents dans l'autre langue. Or, une telle entreprise est quasi impossible puisqu'il faudrait, pour la réaliser, confectionner une série de situations plus ou moins diversifiée, voire illimitée, pour chaque forme proverbiale, d'autant plus qu'il s'agit ici de dictionnaires bilingues, essentiellement de langue, et dont le souci est moins de préciser les contextes d'emploi que de trouver des équivalents thématiques.

Il nous faut donc revenir d'abord sur le sens du proverbe afin de vérifier la proverbialité des dits équivalents et de dégager, à partir de ce sens, un critère qui permettrait à la fois d'apprécier le degré d'équivalence et de servir, dans un second temps, à délimiter le foyer de stéréotypie dans l'énoncé proverbial. Une telle démarche nous permettrait également d'interroger les écarts entre les deux univers linguistiques afin de reposer sur terre le halo d'un énoncé proverbial atemporel et d'une vérité absolue et, ainsi, le réajuster à sa dimension première en tant qu'une certaine représentation parmi d'autres possibles.

\section{L'équivalent proverbial est-il toujours un proverbe?}

Le critère thématique semble être déterminant dans le choix des éléments linguistiques, contextuels et culturels caractérisant les équivalents fonctionnels en langue d'arrivée. Or, ce critère de rapprochement est trop général pour garantir la conser- 
vation des propriétés formelles et sémantiques du proverbe. Autrement dit, l'équivalent ne correspondrait pas, dans certains cas, aux critères définitoires du proverbe par tel aspect ou tel autre.

Sur le plan formel, J. C. Anscombre (2000: 10) établit, entre autres, plusieurs distinctions entre la classe des proverbes et d'autres entités qui partagent avec eux certaines caractéristiques:

- en tant que phrases génériques typifiantes a priori (du type Les voitures ont quatre roues), les proverbes se distinguent des analytiques (Les baleines sont des mammifères) et des typifiantes locales, idiomatiques ou non (comme Quand il n'y en a plus, il y en a encore), qui sont épisodiques et non génériques;

- dans le cadre des phrases génériques, il distingue «les phrases L-sentencieuses (maximes, sentences, apophtegmes, etc.) qui sont des typifiantes locales ayant «un énonciateur premier spécifique» et admettant un combinaison du type "comme l'a dit $X »$ et «les phrases ON-sentencieuses», des phrases typifiantes a priori ayant un ON-énonciateur premier mais indéfini acceptant «la combinaison avec Comme on dit, comme le dit la sagesse des nations, etc.».

Vus sous cet aspect, certains équivalents parémiques en L2 se distinguent nettement des proverbes tels qu'ils sont définis par Anscombre. Les cas les plus clairs concernent des versets coraniques qui transgressent le critère de l'énonciateur premier indéfini:

1- Qui donne aux pauvres, prête à Dieu

Wa-qridu-llaha qardan hasanan (El-Kamil El-Kabir)

Et prêtez à Dieu un emprunt bon

2- Le soleil luit pour tout le monde

kullukum lia:dama wa a:damu min tura:bin tous vous à Adam et Adam de terre (El-Kamil El-Kabir) Vous revenez tous à Adam et Adam est créé de l'argile

Le caractère sacré de ces énoncés bloque formellement leur passage au statut de proverbe contrairement à d'autres d'origine poétique, par exemple, dont l'énonciateur premier spécifique n'est plus connu de la plupart des locuteurs:

3- Le sage entend à demi-mot

Parsil haki:man wa la: tusihi² (El-Marjâ̂)

Envoie un sage et ne le conseille pas

Un autre cas plus problématique se présente avec des équivalents au sens général strictement informatif et littéral et qui n'ont pas les propriétés prosodiques et rythmiques du proverbe comme dans:

4- Qui dort, dîne

annawmu riðc:?un li-zismi (El-Kamil El-Kabir)

Le sommeil (est) nourriture pour le corps.

Si l'on sait que «nourriture» est ici employé d'une manière figurée dans le sens de «bénéfique», la phrase peut être considérée, dans une certaine mesure, comme analytique.

Sur le plan sémantique, G. Kleiber définit le proverbe comme «une unité polylexicale codée, possédant à la fois une certaine rigidité ou fixité de forme et une certaine "fixité" référentielle ou stabilité sémantique, qui se traduit par un sens préconstruit, c'est-à-dire fixé par convention pour tout locuteur, qui fait donc partie du 
code linguistique commun». Il permet de catégoriser, ranger ou rassembler «dans la catégorie dont il est dénomination des occurrences particulières qui le vérifient» (Kleiber 2000 : 40).

À part le caractère figé et les propriétés formelles évoquées ci-haut, deux conditions essentielles sont requises pour qu'une phrase générique soit considérée comme proverbe: l'applicabilité à l'humain et l'existence d'une structure implicative d'ordre inférentiel et non littéral.

De la première condition découlent deux restrictions qui excluent certaines phrases génériques de la classe des proverbes:

- le sens de la phrase étant ou prétendant au statut de proverbe ne peut pas «se réduire à l'attribution d'un prédicat à la classe des hommes» et donc, il ne pourrait être représenté «par une proposition universelle telle que:

$\forall \mathrm{x}$, x est un homme $\rightarrow \mathrm{x}$ est mortel» (Kleiber 2000: 48).

- ce sens ne peut pas également se réduire «à l'attribution d'un prédicat à une sous classe établie d'hommes et il ne peut être représenté par une proposition universelle du type de:

$\forall \mathrm{x}, \mathrm{x}$ est un instituteur $\rightarrow \mathrm{x}$ gagne beaucoup d'argent» (Kleiber 2000: 48).

La deuxième condition exige l'existence d'un sens implicatif qui «n'a pas à être celui du sens littéral ${ }^{3} »$ (Kleiber 2000: 51) et qui est sémantiquement rendu par des structures conditionnelles ayant une formulation du type:

C'est un homme ou les hommes, s'il(s) SV, alors...

L'application de ces deux critères aux équivalents fournis en L2 exclut de la classe des proverbes au moins trois types de phrases génériques.

D’abord, il y a celles qui attribuent une prédication, donnée, à Dieu comme dans:

5- Homère lui-même sommeille quelquefois alYismatu lillehi wahdah (El-Marjâa) l'infaillibilité (est) à Dieu seül

5'- 3elle men le: jeshu: (El-Marjâ̂) est divin celui non oubli Gloire à Dieu qui seul ne peut oublier

6- Un chien regarde bien un évêque alYadamatu lilleh (toute) la gloire (est) à Dieu

Dans toutes ces formules, l'ensemble antécédent et prédication, pris dans le sens littéral, renvoie à des qualités divines. Toutefois, leurs emplois contextuels rattachés à des conduites humaines donnent lieu à des effets inférentiels du type l'homme n'est pas infaillible (5) et (5'), l'homme est fragile. L'absence de structures conditionnelles a pour résultat l'absurdité des propositions universelles telles que:

$\wedge_{\mathrm{xHx}} \mathrm{x}$ est un homme $\rightarrow \mathrm{x}$ est infaillible, fragile comparé à Dieu

L'abstracteur de classe $\wedge^{\wedge} \mathrm{xHx}$ se lit (cf. Kleiber 2000: 49-50) la classe générique des hommes vérifie collectivement le prédicat (x est un homme $\rightarrow \mathrm{x}$ est faillible, fragile comparé à Dieu); autrement dit, les hommes, s'ils sont des hommes sont faillibles, fragiles comparés à Dieu. 
Puis, il y a les équivalents qui attribuent, explicitement, une prédication à la classe des hommes comme dans cette formule qui, à l'origine, provient d'un hémistiche de vers célèbres:

7- Des goûts et des couleurs, on ne discute pas

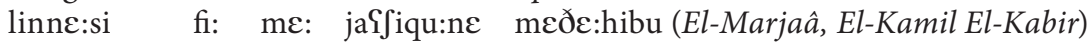

Pour les gens dans ce qu'(ils) adorent des tendances

Les gens ont des préférences différentes

Le sens implicatif du proverbe français peut être quelque chose du genre Les hommes, s'ils ont des goûts différents des nôtres, alors il ne faut pas en discuter. L'équivalent arabe se distingue d'une part par un point de vue sur les préférences en général et d'autre part par l'absence d'une implication explicite portant sur l'attitude qu'on devrait avoir face à cette différence. La structure explicative et informative rend intenable l'interprétation de l'expression par le biais d'une inférence implicative du type ${ }^{\star}$ Les hommes, s'ils ont des préférences différentes, alors il faut les respecter. L'emploi contextuel confirme en partie le caractère aléatoire de ce type d'inférence; l'expression est en effet employée dans deux contextes fort différents: ou bien comme une incitation à la tolérance et, dans ce cas, l'inférence rejoint celle du proverbe français tout en tenant compte de son caractère général; ou bien comme une expression d'étonnement ou même d'indignation face à une attitude ou un comportement qui semblent être excessifs. Par exemple, elle convient bien à exprimer le fait qu'on s'étonne devant quelqu'un qui aime ou qui a l'habitude de manger les insectes.

Un autre cas plus problématique peut être illustré à partir de ce proverbe:

\section{8- L'homme est un loup pour l'homme alPinse:nu Yeduwwu nefsihi (El-Kamil El-Kabir) l'homme (est) l'ennemi de soi-même}

Le proverbe français ne véhicule pas une structure conditionnelle mais il peut être interprété comme donnant lieu à une inférence implicative d'ordre causal du type L'homme incarne le mal pour ses semblables, alors méfie-toi de tes semblables. Faut-il alors exclure de la classe des proverbes les formules ayant ce type d'implication?

Du côté de l'équivalent arabe, la différence actancielle faisant de l'homme à la fois l'antécédent et l'objet restreint le sens dans un cadre strictement informatif. L'implication conditionnelle est alors anomale: ${ }^{\star}$ L'homme, s'il est homme, alors il est ennemi de lui-même.

Enfin, le prédicat peut concerner une sous classe des hommes. L'exemple qui suit montre vraisemblablement les limites de la validité de cette restriction telle qu'elle est définie par Kleiber:

\section{9- Les femmes sont le piège des démons

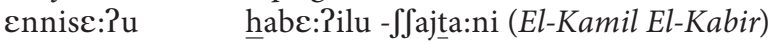 les femmes (sont) les pièges du démon}

Le sens implicatif donnerait une proposition universelle intenable du genre: Les hommes, s'ils sont de sexe féminin, alors ils sont source du mal. La généricité porte ici sur une sous classe stable des hommes; l'abstracteur de classe serait alors ${ }^{\wedge} \mathrm{x} F \mathrm{~F}$ et non ^x Hx. Là encore, la structure conditionnelle n'est pas une obligation. Selon les contextes, le sens peut être interprété comme étant simplement informatif ou implicatif. 
De tout ce qui précède, on peut conclure que si le critère thématique adopté dans les traductions lexicographiques donne lieu à des formules qui ne s'ajustent pas forcément aux critères définitoires du proverbe et qui, par certains aspects, s'écartent du sens original, il est néanmoins justifié par le recouvrement partiel des deux formules dans certains contextes compte tenu des différences culturelles. Il serait donc utile d'étudier ces variations sémantiques du point de vue des différences actancielles puis de celles liées aux schèmes implicatifs afin de délimiter le foyer essentiel de la stéréotypie proverbiale.

\section{Quelles implications pour les différences actancielles entre le proverbe original et son équivalent?}

Au-delà de la parenté thématique plus ou moins présente entre les proverbes français et leurs équivalents arabes, les différences actancielles perçues sur le plan phrastique sont souvent dues à un passage du littéral au figuré, du concret à l'abstrait, du général au particulier, et vice-versa. Pour délimiter l'effet de ces variations, nous avons à saisir leurs impacts sur le plan des actants impliqués, du schéma d'arguments véhiculé par la structure conditionnelle appliquée au sens littéral et du produit de l'abstraction de cette structure qui n'est que «l'implication postulée... responsable de [la] stéréotypicalité » (Kleiber 2000 : 55). Cette manière de procéder vise à vérifier si tout changement à un niveau donné est hérité par le niveau lui succédant en le dotant d'un effet de sens particulier. Ainsi, on pourrait également vérifier si toute différence actancielle se traduit par une différence de la représentation stéréotypique au niveau du schème implicatif abstrait.

Étant donné que «le sens implicatif [du proverbe] n'a pas à être celui du sens littéral» (Kleiber 2000: 55), nous supposons que la participation des actants à ce sens diffère selon qu'il est déduit directement du sens littéral du proverbe ou qu'il est le produit d'une métaphorisation, d'une abstraction ou une montée hypo-hyperonymique (cf. Kleiber 2000: 55). Pour cela, nous regroupons les proverbes et leurs équivalents selon qu'ils conservent le caractère littéral ou figuré ou qu'il y ait passage du littéral au figuré et vice versa.

Le premier groupe peut être illustré par les deux types d'exemples suivants:

10- Secret de deux, secret de Dieu; secret de trois, secret de tous a- sadru-lYa:qili sundu:qu sirrihi (El-Marjâ̂) cœur du sage caisse de son secret

b- sadruka iewsefu lisirrike (El-Marjâ̂) ton cœur (est) plus vaste pour ton secret

c- sudu:ru- liahra:r qubu:ru- lRasra:r (El-Marjaâ) les cœurs des dignes (sont) des tombeaux (pour) les secrets

11- Qui aime bien, châtie bien a- men Peddebe weledehu sari:ran surra bihi kebi:ran (El-Marjâa) qui châtie son fils petit (sera) content de lui grand Qui réprimande son fils à l'âge jeune, en sera fier à l'âge adulte

b- nifme- lmu?eddibi-liasa: (El-Marjâ̂) meilleur éducateur le bâton Le bâton est le meilleur moyen pour éduquer 
D'abord, faut-il souligner que, dans (10), le proverbe français renferme deux propositions conditionnelles correspondant à deux implications complémentaires pouvant être ramenées à une seule générique:

- L'homme, s'il partage son secret avec une autre personne, son secret sera gardé

- L'homme, s'il partage son secret avec deux autres personnes, son secret sera dévoilé

- L'homme, s'il partage son secret avec plus d'une personne, son secret sera dévoilé.

Pour les équivalents arabes, même si les mots «sundu:q» et «qubu:r» sont employés métaphoriquement, le sens du proverbe est toujours compositionnel. La structure conditionnelle est la même que celle du proverbe original: la condition rend compte du nombre de personnes concernées par un secret et la prédication, du résultat disant si ce secret est gardé ou non:

- si tel nombre de personnes est concerné par un secret, alors ce secret sera/ne sera pas dévoilé.

Toutefois, la différence des actants est perceptible dans la condition. Dans (10), il s'agit successivement de «deux» ou "trois personnes»; dans (10a et b), il s'agit de «soi-même» et dans (10c), «les dignes» (le nombre des personnes est indéterminé mais sujet toutefois à une restriction conditionnelle: le fait d'être dignes).

Dans tous les cas, le sens implicatif est sensible à toute variation des actants:

10- L'homme, s'il partage son secret avec plus d'une personne, son secret sera dévoilé $10 \mathrm{a}$ et b-L'homme, s'il garde son secret à lui seul, alors son secret ne sera pas dévoilé 10c- L'homme, s'il partage son secret avec des hommes dignes, alors son secret ne sera pas dévoilé.

Ceci s'explique par le fait que, dans le cadre de la même structure conditionnelle d'un énoncé proverbial à sens compositionnel, chaque actant a son apport sémantique propre qui figure dans la synthèse exprimée dans le sens implicatif.

Au-delà de cet aspect plus ou moins formel, le fait d'avoir la même thématique, la même structure conditionnelle et des sens implicatifs différents, s'explique par la différence des univers de croyance au sein de la même langue ${ }^{4}$ ou entre deux langues différentes (cf. troisième paragraphe).

Pour (11), la différence fondamentale réside dans la variation de la structure conditionnelle:

11- L'homme, s'il aime bien, alors il châtie bien

11a- L'homme, s'il châtie son fils petit, alors il en sera fier une fois grand

11b- L'homme, s'il veut éduquer, alors il doit utiliser le bâton.

De la corrélation amour-châtiment sur laquelle se fonde la structure conditionnelle de départ (condition: amour, prédicat: châtiment), seul le châtiment est retenu dans les équivalents arabes (figure comme condition dans b et prédicat dans c avec une variation de la condition).

De plus, au sens général de «châtier» dans le proverbe en L1, correspondent des spécifications sémantiques qui portent dans (11a) sur l'objet (le fils) et dans (11b) sur le moyen (le bâton). Toutes ces différences font que le recouvrement sémantique entre le sens du proverbe et ceux de ses équivalents est nettement très faible. On conclut de ce fait que, dès qu'il y a une différence d'ordre structurel, on n'est plus face à une diversité de représentations pour un même référent contextuel, mais face à des réfé- 
rents situationnels distincts dont les représentations se croisent d'une manière locale et contingente.

Le second groupe concerne les proverbes et les équivalents fondés sur un mécanisme de figuration ou donnant lieu à un emploi métaphorique du proverbe (cf. Kleiber 2000: 47-48). Nous en donnons respectivement les deux exemples suivants:

12- Les petits ruisseaux font les grandes rivières

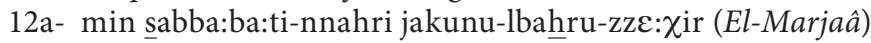
des deltas du fleuve (sera) la mer tumultueuse

12b- mine-lhabbati ten $\int \varepsilon ? u-\iint a z a r a(E l-K a m i l$ El-kabir) du grain pousse l'arbre

13- Tel père, tel fils

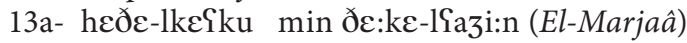
ce gâteau (est) de cette pâte

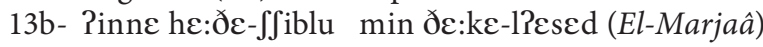
ce lionceau (est) de ce lion

13c- -?el-Yasa: mine-lYusajja (El-Marjâ̂) le bâton (provient) de la tige

Sur le plan phrastique, chaque groupe d'exemples renferme une différence des actants et une similitude du noyau prédicatif verbal qui, respectivement dans (12), (12a) et (12b) et (13), (13a), (13b) et (13c) peut être rendu par les verbes de sens général et abstrait /donner lieu à/ et /provenir de/:

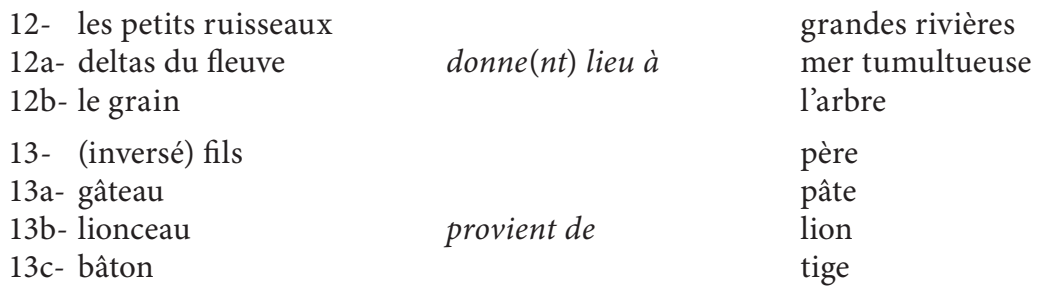

On pourrait remarquer que les deux verbes abstraits sont substituables dans les deux ensembles à condition d'inverser la position des actants:

12- grandes rivières

12a- mer tumultueuse

12b- l'arbre

les petits ruisseaux

deltas du fleuve

13- (inversé) père le grain

13a- pâte

13b- lion

provien(nen)t de

fils

13c- tige

gâteau

donne lieu à

lionceau

bâton

Cependant, si l'on retient le même noyau prédicatif (/provenir de/, par exemple), on se rend compte que le lien sémantique entre la source et le produit n'est pas le même. Dans l'ensemble (12), c'est l'opposition sémique «grand/petit» qui caractérise la relation respectivement entre rivière/ruisseau, mer tumultueuse/deltas du fleuve, arbre/grain. En outre, ce qui est mis en saillance dans la prédication, c'est l'insignifiance de l'objet indirect (la source) par rapport au sujet (le produit).

Dans (13), à une exception près (tige/bâton), on assiste à la même opposition sauf que l'inversion des actants fait figurer l'axiologiquement positif dans le prédicat (père, lion, bâton) et porte la saillance sur les qualités de la source ou disant pour le moment sur la source tout court. 
Cette différence a une incidence apparente sur le sens implicatif. Dans les exemples en (12), le contraste entre l'insignifiance ou la petitesse de la source et la grandeur du produit permet d'inférer immédiatement le sens du proverbe qui pourrait être quelque chose comme:

- Avec le temps, l'homme, s'il prend soin de ce qui lui paraît peu ou insignifiant en sa possession ou à sa disposition, alors il obtiendra, comme fruit, un résultat important.

Par contre, dans les exemples en (13), le contraste en lui-même n'exprime que le lien de parenté (biologique ou matérielle) entre la source et son "produit». L'essentiel ne peut être déduit qu'à partir de ce qui est mis en saillance: les qualités de la source. L'inférence implicative pouvant être exprimée par un noyau prédicatif du type /ressembler/ est indirecte:

- L'homme, s'il engendre une création, alors celle-ci sera à son image/lui ressemblera.

Dans les deux types d'exemples, nous voyons que la différence actancielle n'a pas d'incidence directe sur le sens implicatif résultant d'une abstraction implicative ou d'un emploi métaphorique du proverbe à sens littéral.

Passons maintenant au troisième groupe d'énoncés proverbiaux où il y a passage du littéral au figuré. Les deux exemples suivants nous serviront d'illustration:

14- Qui se ressemble, s'assemble

14a- $\varepsilon$ ttuju:ru fele: $1 \varepsilon \int k \varepsilon: l i h \varepsilon: \quad$ teqaSu (El-Kamil El-kabir, El-Marjâ̂) les oiseaux sur leurs semblables se posent

15- Qui aime bien, châtie bien

15a- -alliq sawteke hejou jara:hu Pehluke (El-Marjâ̂) attache ton fouet là où (il) sera vu (par) ta famille [ta femme].

15b- darb-lhabi:b zbi:b (El-Marjâ̂) le châtiment de (la part) du bien-aimé (est) du raisin sec Le fait d'être châtié du bien-aimé est un plaisir

Dans les exemples en 14, le sens littéral est:

- Si les oiseaux se ressemblent, alors ils se rassemblent.

Le sens implicatif ne fait que substituer hommes à oiseaux. Donc, l'actant « oiseaux» n'est qu'une valeur de la variable $\mathrm{x}$ dans le schème abstrait si des $x$ se ressemblent, alors ils s'assemblent et, de ce fait, n'a aucune incidence sur le sens du proverbe et de ses équivalents tant que la structure conditionnelle est la même dans les deux cas.

En revanche, dans les exemples en (15), nous avons le même cas que les exemples en (11): la différence ne concerne seulement pas les actants mais toute la structure conditionnelle:

15a -sens littéral: L'homme, s'il veut que sa famille craint son fouet, alors il doit l'attacher en un lieu apparent

- sens implicatif: L'homme, s'il veut que sa famille lui obéisse, alors il doit se faire craindre.

Comme dans (11), la condition est différente et la prédication ne retient que le thème du châtiment présenté non comme un fait mais comme une simple menace. De ce fait, là encore, c'est la différence de la structure qui est responsable de la différence sémantique entre le proverbe et ses équivalents; les actants ne font que suivre. 


\section{5b- L'homme, s'il châtie par amour, alors son acte sera adoré}

Le sens du proverbe exprime en quelque sorte le point de vue de celui ou plutôt de celle qui subit le châtiment. Ici, l'ensemble de l'antécédent et de la prédication d'origine figure dans la condition de l'équivalent; le tout donne lieu à une nouvelle implication (son acte sera adoré). Comme dans l'exemple précédent, la différence structurelle se traduit par une différence de l'inférence implicative malgré la similitude relative des éléments prédicatifs (la corrélation entre amour et châtiment est présente dans les deux proverbes).

De tout ce qui précède, on peut résumer que l'effet de la différence actancielle est manifeste dans les cas où le proverbe et ses équivalents ont un sens littéral. Dans les cas où il y a passage du figuré au figuré ou du littéral au figuré, c'est la structure sémantiquement structurelle qui est déterminante dans la synthèse sémantique: si elle est la même, la différence des actants n'a pas d'effet direct sur le sens implicatif; si elle n'est pas la même, ce sens change même s'il y a une certaine identité entre les éléments prédicatifs.

Reste maintenant à voir dans quelle mesure «l'implication [ainsi] postulée est responsable de la stéréotypicalité» (cf. Kleiber 2000: 55). L'étude contrastive des implications inférées par certains proverbes et leurs équivalents nous permettrait de répondre à cette question.

\section{Sens implicatif et univers de croyance}

Comme le montre Kleiber (2000: 55), la structure conditionnelle («le schème implicatif $[+\mathrm{H}] »)$ ne suffit pas pour ériger en proverbe un énoncé ayant formellement un revêtement parémique. Il avance l'exemple suivant:

- Il faut se laver les mains avant de manger.

qui tout en admettant l'analyse selon le modèle implicatif des proverbes (si on veut manger, il faut se laver les mains avant) «ne peut revêtir une livrée parémique, parce que, précisément le fait de se laver les mains n'est pas saisi comme une conséquence logique qui découle du fait de manger: on peut fort bien manger sans se laver les mains au préalable!» (Kleiber 2000: 55). Cette assertion nécessite d'être nuancée puisque ce n'est pas parce qu'on peut bien manger sans se laver les mains qu'il n'y a pas de stéréotypie. Certes, il faut qu'il y ait « une connexion stable» entre la condition énoncée et la conséquence qui en découle (cf. Riegel 1987: 95, cité par Kleiber) mais doit-on également préciser que tout proverbe est stéréotypique justement parce qu'il existe une alternative à la conséquence logique qu'il consacre. Ainsi, une phrase analytique à une seule implication ne peut donner lieu à un proverbe:

16- Un mort ne revient jamais à la vie

- L'homme, s'il est mort, alors il ne reviendra jamais à la vie

D’ailleurs, les proverbes ayant un sens littéral fondé sur ce type d'implication unique connaissent forcément "une montée abstractive de type hypo/hyperonymique» (Kleiber 2000: 55). Tel est le cas dans le proverbe arabe suivant:

17- me: darra- $\iint \varepsilon: t \varepsilon \quad b \varepsilon f d \varepsilon$ ocbhihe: qu'est-ce qui nuit à une brebis après l'avoir égorgée?

- Qu'est-ce qui pourrait nuire à la brebis une fois égorgée? 
Le fait d'appliquer le schème implicatif aux humains tout en maintenant le thème de la mort et de la nuisance physique ne suffit pas pour ériger la proverbialité de l'énoncé:

- L’homme, s'il meurt, alors les sévices ne lui nuisent plus

Pour qu'il ait un sens proverbial, il faut envisager un degré plus élevé d'abstraction qui, dans ce cas, projette le drame de la mort dans le domaine de la morale:

- L’homme, si sa réputation est touchée, alors aucun autre malheur ne compte pour lui

$\mathrm{Au}$-delà de cette conséquence stable et communément admise puisque figée, la porte reste ouverte dans les faits pour d'autres implications:

- L’homme, si sa réputation est touchée, alors il ne faut pas le pousser plus loin dans le désespoir/il faut l'aider à supporter son malheur.

De ce fait, le sens implicatif d'un proverbe est censé être vérifié par un ensemble de référents situationnels idiosyncrasiques mais qui forment une catégorie référentielle de scénarios de portée générale que rend le caractère générique de la phrase proverbiale. Autrement dit, l'aspect gnomique du proverbe fait de lui une devise qui conviendrait à toute situation ayant la caractéristique $\mathrm{x}$. Or, dans les faits, certaines de ces situations échappent d'une manière ou d'une autre à la rigidité de l'implication proverbiale et peuvent même donner lieu à des énoncés proverbiaux contradictoires (cf. note 5). De là provient la donne stéréotypique puisque la même catégorie de situations donne lieu à plus d'une implication possible alors que le proverbe n'en retient qu'une.

L'approche contrastive est fort utile pour illustrer ce phénomène puisqu'elle nous épargne de chercher parmi une infinité de contextes celui qui infirme la devise proverbiale et révèle certains aspects de sa stéréotypicalité.

Nous avons vu que, a priori, tout proverbe est construit autour d'une structure conditionnelle qu'on peut analyser en condition et implication de la condition. La catégorie référentielle des situations particulières auxquelles le sens proverbial devrait convenir est rendue par la condition. L'implication, elle, exprime toujours une inférence univoque par rapport à cette catégorie. En elle-même, elle ne peut être le foyer de la stéréotypie puisque le stéréotype présente toujours une certaine représentation de quelque chose, d'un fait, alors que l'implication est une inférence à partir de ce fait et on ne peut pas en toute logique avoir une représentation d'une implication qui est elle-même inférée. Donc, la stéréotypie provient de la corrélation stable entre une situation donnée et l'inférence implicative qui en découle.

Cet a priori stéréotypique est tributaire de la diversité des univers de croyance. Entre deux langues telles que l'arabe et le français les charges stéréotypiques portées par les proverbes peuvent aller de la simple différence de degré à un découpage différent du faisceau de référents situationnels impliqué par le proverbe, à l'opposition nette entre les représentations.

Les exemples suivants illustrent le premier type de différences:

18- Le sage entend à demi-mot

18a- Persil hakki:man we le: tusihi (El-Marjâ̂) envoie un sage et ne le conseille 
19- Petit à petit, l'oiseau fait son nid

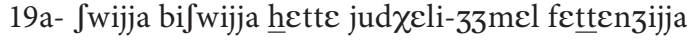
petit à petit jusqu'à ce que entre le chameau dans la marmite C'est petit à petit qu'on finit par introduire le chameau dans la marmite (Dictionnaire bilingue des proverbes marocains)

20 - Faute avouée est à demi pardonnée

20a- PelPiYtira:fu bil- $\chi$ ata?i fad:la (El-Kamel El-Kabir) la reconnaissance de la faute (est) une vertu

20b- lli: qar bðenbu: yferlu: rabbi: celui qui reconnaît sa faute sera pardonné par Dieu (Dictionnaire bilingue des proverbes marocains)

Dans tous ces exemples, la condition exprimée par le proverbe original et ses équivalents est identique alors que les conséquences logiques sont légèrement différentes:

18- L'homme, s'il est sage, alors il comprendra à demi-mot

18a- L'homme, s'il est sage, alors il comprendra sans mot aucun

19- L'homme, s'il ménage ses petits efforts, alors il fait de bons résultats

19a- L'homme, s'il ménage ses petits efforts, alors il parvient à réaliser l'impossible

20- L'homme, s'il reconnaît sa faute, alors il est à demi pardonné

20a- L'homme, s'il reconnaît sa faute, alors il son acte sera vertueux

20b- L'homme, s'il reconnaît sa faute, alors Dieu le lui pardonne ${ }^{5}$.

Respectivement, entre à demi mot/sans mot aucun, bons résultats/l'impossible, à demi pardonné/acte vertueux/ pardonné, la différence est une affaire de degré.

Le second type de différences concerne le découpage de l'espace référentiel auquel renvoie l'énoncé proverbial. À:

21- La faim fait sortir le loup du bois.

correspond, en arabe, un paquet d'équivalents:

21a- addaru:ra:t tubi:hu-lmahðu:ra:t (El-Kamel El-Kabir, El-Marjâ̂) les nécessités tolèrent les interdits

21b- bez3u:S kejarda $\varepsilon$ sbef bezzi:fe

à cause de la faim accepte le lion la charogne

C'est à cause de la faim que le lion accepte de manger la charogne

(Dictionnaire bilingue des proverbes marocains)

21c- elberd kajfellem sri:qt el-fhem

le froid apprend le vol du charbon de bois

Le froid apprend à voler le charbon de bois (Dictionnaire bilingue des proverbes marocains).

Les sens implicatifs respectifs se distinguent sur le plan de la conséquence:

21- L'homme, s'il vit dans le besoin, alors il devient imprudent

21a- L'homme, s'il vit dans le besoin, alors il transgresse l'interdit

21b- L'homme, s'il vit dans le besoin, alors il s'humilie en acceptant ce qu'il y a de plus répugnant.

21c- L'homme, s'il vit dans le besoin, alors il s'intéresse même aux choses de peu de valeur.

Chaque proverbe focalise sur un aspect particulier de la conduite humaine (à part (21a) qui a une portée plus ou moins générale). Cependant, la similitude de la condition dans tous les proverbes n'est qu'illusoire (L'homme, s'il vit dans le besoin). Autrement dit, aucun proverbe ne convient à toutes les situations impliquées par les 
autres et dans lesquelles l'être humain se trouve en état de nécessité. La raison est que chaque implication sélectionne un type particulier de contextes qui ne vaut pas pour les autres. Si par exemple un homme pauvre descend dans la rue pour mendier, seuls (21b), et à un degré moindre (21a), conviennent pour rendre compte de cette situation.

Enfin, le troisième type de différence concerne des représentations partiellement ou totalement contradictoires:

22- Loin des yeux, loin du cour.

22a- tu:lu- ttenc:?i: mesle:tu-ttesasa:fi: ${ }^{6}$ (El-Marjâ̂) la durée de l'éloignement (annonce) le plaisir de l'entente

23- des goûts et des couleurs, on ne discute pas

23a-kul Yle: ki:fek wi-lbis Yle: ki:f enne:s mange selon ton goût et habille-toi selon le goût des gens (Dictionnaire bilingue des proverbes marocains)

Malgré la similitude relative de la condition dans (22) et (22a), les conséquences sont tout à fait contradictoires:

22- L'homme, s'il reste loin de ceux qu'il aime, alors ses sentiments s'affaiblissent.

22a-L'homme, s'il reste loin de ceux qu'il aime, alors l'entente sera forte lors de la rencontre.

Dans les exemples en (23) et (23a), les deux actants figurant dans la condition dans le proverbe français (goûts/couleurs) sont dissociés dans l'équivalent qui réserve à chacun d'eux une implication propre, l'une se rallie au sens du proverbe original (goûts) et l'autre s'y oppose (couleurs).

D’une manière générale, chaque langue, en harmonie avec l'univers de croyance de ses locuteurs et de la vision du monde qu'elle véhicule, sélectionne, parmi $n$ conséquences logiques découlant d'un type de situations donné, une ou plusieurs implications qu'elle associe, dans le cadre de l'énoncé proverbial, à cette catégorie de contextes. De ce fait, la sagesse des peuples privilégie, parfois différemment, une vérité parmi d'autres possibles qui serait adoptée par toute la communauté linguistique.

Les lexicographes bilingues travaillant sur les proverbes devraient, en principe, être guidés dans la recherche des équivalents par l'invariant contextuel et situationnel quand celui-ci ne fait pas l'objet d'un découpage différent comme dans les exemples en (21) et (23). Dans les faits, on constate une divergence des pratiques selon la vocation des dictionnaires. Ceux de la langue générale optent plutôt pour le critère thématique général: tout invariant de quelque type qu'il soit, situationnel ou inférentiel, suffit pour établir la connexion entre les énoncés proverbiaux dans les deux langues. Par contre, certains dictionnaires bilingues spécialisés dans les proverbes (par exemple Le dictionnaire bilingue des proverbes marocains) associent traduction littérale, indices sur l'emploi contextuel et équivalents dans d'autres langues, d'où la plus grande parenté entre les proverbes originaux et leurs équivalents. 


\section{Conclusion}

L'approche contrastive des proverbes s'avère intéressante au moins à trois titres:

1- sur le plan de la traduction, elle a permis de:

- vérifier le degré de proverbialité des équivalents à partir de certains critères formels et sémantiques. Il a été démontré dans ce sens que les dictionnaires bilingues sont parfois insensibles à la différence qui existe entre des formes sentencieuses fort éloignées (cf. par exemple (1), (2) et (4);

- montrer les limites du critère thématique dans le choix des équivalents (cf. les exemples en (11) et (15). Nous avons trouvé que la configuration de la structure conditionnelle est un indice important permettant de juger du degré de l'équivalence proverbiale: l'équivalent parfait est celui qui a la même condition formulée dans l'énoncé d'origine.

2- sur le plan du proverbe lui-même, cette approche a permis de:

- tester certains critères sémantiques définitoires du proverbe à partir d'une langue autre que le français. Des cas problématiques ont besoin d'être éclairés davantage (cf. l'exemple en (9) concernant l'impossibilité pour une sous classe des hommes d'être un antécédent de la structure implicative du proverbe);

- analyser l'impact de la différence actancielle sur le sens implicatif abstrait. Nous avons pu vérifier que, quand la structure conditionnelle est la même dans deux énoncés proverbiaux, la variation des actants n’a de conséquences sémantiques sur le sens implicatif que lorsque celui-ci est directement inféré du sens littéral du proverbe.

3- sur le plan de la stéréotypicalité du proverbe, nous retenons les faits suivants:

- la stéréotypie provient de la non-exclusivité d'une inférence implicative associée d'une manière stable à une catégorie de référents situationnels; d'où la notion d'«écart» entre les potentialités inférentielles que pouvaient susciter un type de situations et le caractère générique ou gnomique caractérisant une association particulière véhiculée par l'énoncé proverbial;

- si donc la stéréotypie proverbiale est d'ordre relationnel (entre un type de situations et ce qu'il infère), son foyer est l'implication, objet de la variation; le ou les référents représentés dans la condition étant censés être invariables;

- la même catégorie de référents situationnels pourrait donner lieu à plusieurs implications stéréotypiques (parfois contradictoires) formulées dans plusieurs proverbes partageant, sur le plan structurel, la même condition;

- entre deux langues, le phénomène est plus complexe puisque à cela s'ajoute le fait que l'espace référentiel lui-même n'est parfois ni perçu ni découpé de la même manière.

Tous ces aspects méritent d'être repris en multipliant les perspectives d'analyse et il en reste d'autres. Nous voulons dans ce sens ajouter une autre «pierre» dans le vaste «jardin universel des proverbes » baptisé par Kleiber; c'est celui des restrictions de l'usage. Certains énoncés proverbiaux ont vraisemblablement un sens implicatif abstrait qui s'appliquerait à $n$ contextes particuliers. L'usage n'en privilégie qu'un sous-ensemble. Tel semble être le cas dans les deux proverbes suivants:

24- Les petits ruisseaux font les grandes rivières.

25- $\varepsilon$ ttuju:ru fele: $2 \varepsilon \int k \varepsilon: l i h \varepsilon: \quad$ teqaYu (El-Kamil El-kabir, El-Marjâ̂) les oiseaux sur leurs semblables se posent 
Le proverbe en (24) est «surtout appliqué aux sommes d'argent» (Rey et Chantreau 1997); celui en (25) est exclusivement employé dans des contextes axiologiquement négatifs alors que rien dans leurs structures ne prédit cette orientation.

\section{NOTES}

1. Notre corpus est puisé dans Al-marjaâ (dictionnaire bilingue arabe-français), Dictionnaire bilingue des proverbes marocains (arabe-français) et El-Kamel El-Kabir (français-arabe).

2. À l'origine, il s'agit d'un hémistiche d'un vers de Zoubeir Ibn Abd Elmuttalib:

Piðع: kunta fi: ha:zatin mursilan, fa-rsil haki:man wa la: tusihi

Si tu as besoin d'envoyer un messager, envoie un sage sans le conseiller.

3. «Le facteur implicatif explique dans une perspective gricéenne pourquoi certains proverbes et pas d'autres ont un sens implicatif qui est l'hyperonyme de la situation implicative littérale» (Kleiber 2000: 58).

4. Laspect contradictoire entre (10a et 10b), d'une part, et (10c), d'autre part, traduit la diversité des représentations pour un même référent situationnel et confirme l'écart entre l'aspect gnomique de l'énoncé proverbial et la variété réelle de la manière d'être ou d'être représenté pour un fait donné. Un tel écart est le fondement même de tout phénomène stéréotypique.

5. Pour un besoin de clarté, nous avons omis des équivalents établissant une hiérarchie entre la reconnaissance de la faute et la repentance:

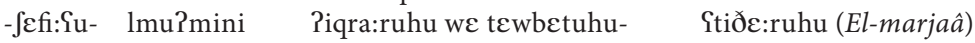

le pardon du croyant (est) son aveu et sa repentance (est) son excuse

- etewbetu tuðhibu-lharbatu (El-marjâa)

la repentance éloigne le poignard (le châtiment).

6. Comme on l'a déjà mentionné dans la note 5 , cet aspect contradictoire des proverbes pourrait exister au sein d'une même langue. Pour ce cas précis, il y a deux équivalents qui ont le même sens que l'énoncé proverbial original:

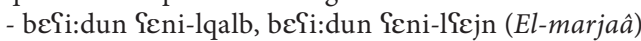

Loin des yeux, loin du cœur

- عlbuidu $3 \varepsilon f \varepsilon: ?$ (El-marjâ̂)

l'éloignement (est un signe de) froideur.

\section{RÉFÉRENCES}

Anscombre, J. C. (2000): «Parole proverbiale et structures métriques», Langages 139, pp. 6-26.

Kleiber, G. (1999) : «Les proverbes: des dénominations d'un type "très très spécial” ", Langue française 123, pp. 52-69.

KLeiber, G. (1999): «Les proverbes antinomiques: une grosse pierre «logique» dans le jardin toujours "universel" des proverbes», in Bulletin de la Société de Linguistique de Paris 94-1, pp. 185-208.

KLeiber, G. (2000): «Sur le sens proverbial», Langages 139, pp. 39-58.

MejRi, S. (1996): «Binarisme, dualité et séquences figées», in Les formes du sens, Mélanges Robert Martin, Duculot, Collection «champs linguistiques», pp. 249-256.

MejRI, S. (1997): Le figement lexical: descriptions linguistiques et structuration sémantique, Publications de la Faculté des lettres de la Manouba, volume X.

MejRI, S. (1998) : «La globalisation sémantique», Neophilologica, T 13, Red. W. Banys, Katowice, pp. 83-93.

MejRI, S. (2001): «La structuration sémantique des énoncés proverbiaux», L'information grammaticale 88 , pp. 10-15.

Mejri, S. (2003): «La stéréotypie du corps dans la phraséologie. Approche contrastive», in Burger, H., Häcki Buhofer, A. et G. Gréciano, Phraseologie und Parömiologie, Band 14, Flut von Texten-vielfalt der Kulturen, Schneider Verlag Hohengehren GmbH, pp. 203-217.

MejRi, S. (2000): «L'écriture littéraire bilingue: traduction ou réécriture? Le cas de Salah Guermadi», Meta 45-3, Presses de l’Université de Montréal, pp. 450-457. 
Michaux, C. (1999): «Proverbes et structures stéréotypées», Langue française 123, pp. 85-104.

TамвA, I. (2000): «Formules et dire proverbial», Langages 139, Larousse, pp. 110-118.

\section{Dictionnaires}

El-Marjâ̂ (2002), Librairie du Liban.

El-Kamel El-Kabir (2001, 4ème édition), Librairie du Liban.

Dictionnaire bilingue des proverbes marocains, V 1, (1997), L'Harmattan. Dictionnaire des expressions et locutions (1997, nouvelle édition), Le Robert. 